\title{
GEOMETRICAL ANALYSIS OF LIGHT-EMITTING DIODE FOR ENHANCING EXTRACTION EFFICIENCY
}

\author{
ADAM SHAARI*1, AHMAD FAKHRURRAZI AHMAD NOORDEN ${ }^{1}$, SAIFUL NAJMEE \\ MOHAMAD² AND SUZAIRI DAUD ${ }^{3}$
}

${ }^{1}$ International Islamic University Malaysia, Kuantan 25200 Pahang, Malaysia. ${ }^{2}$ Universiti Sains Malaysia, Penang, 11800 Malaysia. ${ }^{3}$ Universiti Teknologi Malaysia, Skudai 81310 Johor, Malaysia.

*Corresponding author: adamshaari123123@gmail.com

Submitted final draft: 5 March $2020 \quad$ Accepted: 10 April 2020

http://doi.org/10.46754/jssm.2020.08.006

\begin{abstract}
A non-uniform current spreading in the current spreader can greatly reduce the efficiency of the light-emitting diode (LED). The effects of the electrode contact area to the spreading layer towards extraction efficiency of LED chips is analysed in analytical simulations. Length of current spreading and light extraction efficiency is analysed for variation of contact area. The contact area value is varied by changing the shape of the electrode and the value of width of contact area. The increase in contact area decreases light extraction efficiency as more light are absorbed by the bottom electrode surface. The effective current spreading length for Indium Tin Oxide (ITO) of thickness $300 \mathrm{~nm}$ is $36.44 \mu \mathrm{m}$. The 6 strips 'fork' design is the most optimum. The design has the most area for photons produced in active region to escape without reducing the area cover with current density. This enables the chip to has more extraction efficiency with more uniform current spreading.
\end{abstract}

Keywords: Light-emitting diode, spreading pad, indium tin oxide, extraction efficiency.

\section{Introduction}

Light-emitting diode (LED) has become a preferred research area for its contribution in producing highly efficient and environment friendly light source (Ryu et al., 2011). Increasing the efficiency of LED has been the main concern in the lighting technologies and industries (Hao et al., 2016). The main factor for the reduction of efficiency of LED a non-uniform current spreading in the LED (Wang et al., 2010; Zhou et al., 2017). This is highly dependent on the pattern and configuration of epitaxial-layer of the LED. This research focused on improving current uniformity in ITO and providing design with high extraction efficiency.

Gallium-Nitride-based (GaN-based) LED can have vertical design that stacks every layer on top of another to form epitaxial-layer. The design does improve LED performance significantly as larger vertical current gives lower device resistance. However, the light output was lowered as light emitted was absorbed by the n-electrode (Kim et al., 2007). Electrode pattern improvement was made by Yun et al. in 2009 that improves the current uniformity in mesa-structured GaN/InGaN blue light-emitting diodes (LEDs). Comparisons between an LED with a new electrode pattern taking the proposed methodology and an LED with a commercially used electrode were made by comparing both current and luminance distributions. They verified the effect of the proposed methodology by both simulations and experiments (Yun et al., 2009). Another advancement was made on lateral GaN-based LEDs. The lateral current spreading (CS) effect on the efficiency droop in GaN-based LEDs was studied in terms of the CS distance. The research found a trend of improvement in the efficiency droop of LEDs with shorter CS distances. However, the effect of CS performance on ideality factors, $n_{\text {ideal }}$, of LEDs is not significant (Huang et al., 2013). Zhou et. al has analysed LED with discontinuous n-electrode and reflective p-electrode design on lateral LED. The effect of opaque p-electrode towards light absorption is reduced in this research as the p-electrode was made reflective 
by double $\mathrm{Cr} / \mathrm{Pt} / \mathrm{Au}$ layers (Zhou et al., 2013). Besides, GaN-based flip-chip LED by inserting a silver (Ag) reflector covered with diffusion barrier of tungsten-titanium (TiW) is analysed with a strip-shaped $\mathrm{SiO}_{2}$ current blocking layer design to reduce the effect of current crowding in ITO layer. The fabricated design shows the improvements on current uniformity in ITO layer, light output power and maximum output power of the LED. The Ag reflector increases the optical reflectance while the current blocking improved the current spreading of the LE (Zhou et al.,2017). Silicon Dioxide $\left(\mathrm{SiO}_{2}\right)$ blocking layer is also used to provide better extraction efficiency of the LED by blocking the concentration of current in the area right below the p-electrode and reducing the amount of light absorbed in the p-electrode (Huh et al., 2002 and Zhuo et al., 2015). Later in 2019, Zhuo and his team made an advancement by inserting a high reflectance of $\mathrm{Ag}$ film of $95.0 \%$. The research found that the Ag-based reflector increased the light extraction efficiency (LEE) of the LED and improved the current spreading (Zhuo et al., 2019).However, low current uniformity in the epitaxial layer causes low recombination rate in which reduces the chip efficiency. Big electrode increases the current uniformity but decreases the light extraction efficiency.
In this work, the research simulated improved current spreading across indium tin oxide (ITO) layer while having the design of electrode that does not reduce the extraction efficiency of a vertical LED. The research focused on analytical formulation for design of electrode with the most exposed area for photon escape from the epitaxial-layer for enhanced extraction efficiency.

\section{Materials and Methods}

\section{Indium Tin Oxide}

The experiment considered growing the layer of current spreading pad ITO on $\mathrm{p}-\mathrm{GaN}$ layer for better current spreading to the $\mathrm{p}-\mathrm{GaN}$ layer. The p-type GaN has low conductivity. The resistivity is bigger than $1 \Omega . \mathrm{cm}$ as the hole mobility in III-V nitrides is $1 \sim 20 \mathrm{~cm}^{2} /(\mathrm{V} \mathrm{s})$ and the concentration of hole is in the range of $10^{17}$ $\mathrm{cm}^{-3}$, resulting in resistivity to be bigger than 1 $\Omega \mathrm{cm}$ (Schubert, 2018). The chosen thickness of ITO used in this research was $300 \mathrm{~nm}$ (Li et al., 2012).

\section{Extraction Efficiency}

Photons produced in the active layer must be transmitted to escape into free space from the LED chip. Extraction efficiency can be described as:

$$
\begin{gathered}
\eta_{\text {extraction }}=\frac{\text { number of photon emitted into free space per second }}{\text { number of photon emitted from active region per second }} \\
\qquad \eta_{\text {extraction }}=\frac{P /(h v)}{P_{\text {int }} /(h v)}
\end{gathered}
$$

where is the optical power emitted into free space and is the optical power emitted from the active layer. Not every photon produced in the active layer manage to escape LED chip. This is caused by few factors such as light absorption in the substrate of the LED, absorption by metal electrode and total internal reflection (Schubert, 2018). 


\section{Current Spreading Length}

In conventional vertical LED design, the current is injected directly from the top electrode into the active region through p-type material. This will introduce more current crowding in the p-type material and resulting in low efficiency of the LED. To prevent this from happening, current spreading layer is introduced to spread the current in the p-type material, ensuring a more uniform current across the active region. The absence of the current spreading layer can result in the current-injected area in the active region to be only slightly bigger than the electrode contact area (Schubert, 2018). Current spreading layer used in this experiment is ITO. Equation (3) shows how current density, , is related to distance from the electrode contact area

$$
J(x)=\frac{2 J_{0}}{\left[\frac{x-r_{c}}{L_{s}}+\sqrt{2}\right]^{2}} \quad\left(x \geq r_{c}\right)
$$

where $L_{s}$ is the effective current spreading length, $r_{c}$ is the diameter of the electrode and $x$ the distance in measurement relative to the center of electrode. $L_{s}$ is defined by equation (4) as

$$
L_{s}=\sqrt{\frac{t n_{\text {ideal }} k T}{\rho J_{0} e}}
$$

where is the resistivity of the ITO, is the ideality factor of the chip and is the thickness of the ITO (Schubert, 2018). Table 1 shows the parameters used in this research to obtain the value of $L_{s}$.
Table 1: Parameters for spreading length calculation

\begin{tabular}{cl}
\hline Variable / Parameter & \multicolumn{1}{c}{ Value } \\
\hline $\mathrm{t}$ & $300 \mathrm{~nm}$ \\
& 1.2 \\
$\mathrm{kT} / e$ & $25.9 \mathrm{mV}$ \\
$\mathrm{J} 0$ & 2000000 \\
$\rho$ & $3.51 \times 10^{-6} \Omega . \mathrm{m}$ \\
\hline
\end{tabular}

The model used in this research assumes a constant potential and current density () in the area under the metal $\left(x<r_{c}\right)$. Figure 1 illustrates how current spreading layer can affect the current flow and current density below and electrode.

Equation (3) states that the value of current density decreases with increasing value of distance to the edge of strip. To verify the pattern and compatibility of the equation with the research, an analytical formulation has been made to obtain the pattern of the graph. Equation (4) and the pattern of the graph gives the value of. Figure 1 shows the pattern of current density extending away from the edge of electrode contact. is the value of distance from the edge of electrode where the tangent line to the curve when $x$ is equals to $r_{c}$ intercepts the base of the ITO layer (Schubert et al., 2018). Equation (4) was used to find the in ITO based on the size and pattern of electrode on the ITO layer. The of the ITO determined the size of electrode to effectively spread the current across the whole surface of epitaxial-layer.

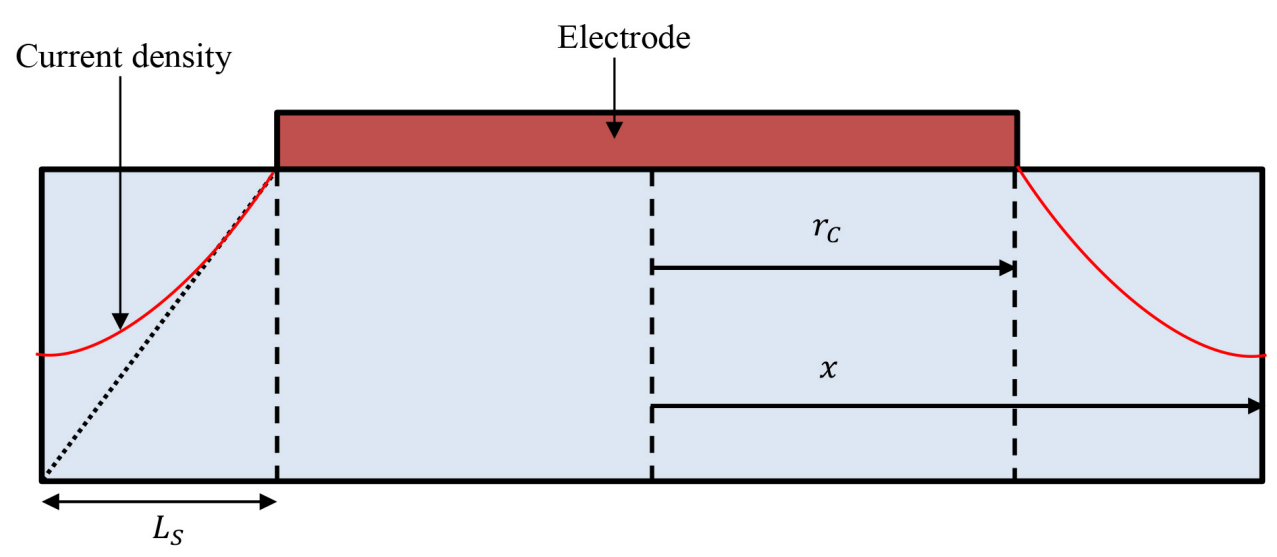

Figure 1: Schematic illustration on how current is spread inside ITO layer 


\section{Electrode}

The pattern electrode is designed to have a shape that can provide sufficient current density across active region without reducing the extraction efficiency of the chip. That was done by having a maximum distance of from the edge of electrode to the edge of epitaxial-layer. Figure 2 explains the pattern of electrode for most uniform current density across active region.

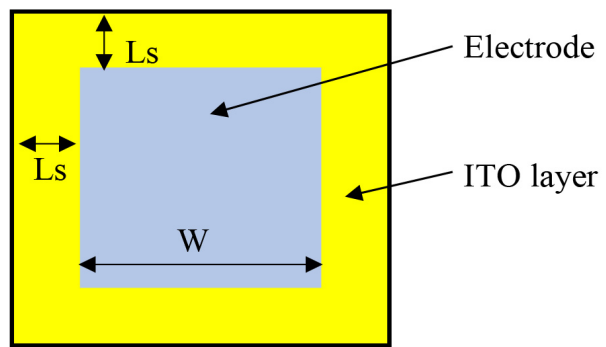

Figure 2: Top view of LED chip for design with uniform current spreading area

The design will give good current uniformity, but will allow low extraction efficiency. To increase the extraction efficiency of the LED, a fork design was proposed. The design will allow current uniformity with less area of electrode in contact with ITO. Figure 3 below explains the fork design with two strips.

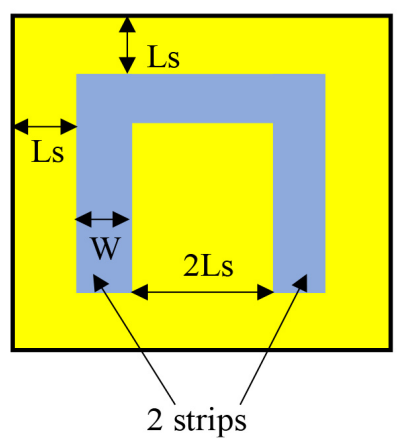

Figure 3: Top view of LED chip with fork design to maximize extraction efficiency

The research focuses on the variation of number of strips of electrode for the fork design. The area of ITO layer not covered by electrode for every variation was analyzed. The design with the biggest area not covered by the electrode is the design with the highest extraction efficiency as it has the most area available for the generated photon in the active region to escape the chip.

\section{Results and Discussion}

\section{Effective Current Spreading Length}

The research focused on finding the effective current spreading length and also the most suitable 'fork' design for maximum exposed area for photon escape. The research found that the value of for ITO of thickness $300 \mathrm{~nm}$ is $36.44 \mu \mathrm{m}$. The value was used to find the area of p-electrode on top of ITO. The value of provided the information needed for width, $W$, of electrode for every variation of number of stripes. The value of $W$ gives the area of electrode in contact with the ITO. The area exposed is found by the difference from the total ITO area to the electrode contact area.

\section{Electrode Design}

Table 2 shows the values of area of electrode and the exposed area for photon to escape for designs with different number of strip.

Table 2 shows that the highest value of exposed area is acquired when the number of electrodes is 6 at width $10.44 \mu \mathrm{m}$. This allows for maximum extraction efficiency and uniform current spreading across the ITO. The lowest value of exposed area is acquired when the number of electrodes is 1 at width of $427.11 \mu \mathrm{m}$. This is because, for single strip electrode, the width has to be at $427.11 \mu \mathrm{m}$ for the current to be uniformly spread across ITO. Figure 5 shows the graph of comparison for value of $\mathrm{W}$, area of electrode in contact and exposed area for photon escape for all 6 variants of number of stripes.

The graphs in Figure 4 show the relation of number of strips to the value of exposed area for light emission. The graphs show that the value of exposed area increases as the number of strips increases. The design with least area of contact of electrode to ITO have the least number of photons produced in the active region absorbed 
Table 2: W, Area of electrode and area exposed for photon escape for every variation of number of stripes in each fork design.

\begin{tabular}{cccc}
\hline $\begin{array}{c}\text { Number of } \\
\text { strip }\end{array}$ & $\begin{array}{c}\text { Width of strip, } \boldsymbol{W} \\
(\mu \mathrm{m})\end{array}$ & $\begin{array}{c}\text { Area of electrode in contact } \\
\text { with } \mathbf{I T O}\left(\mathbf{m}^{2}\right)\end{array}$ & $\begin{array}{c}\text { Area exposed for photon } \\
\text { escape }\left(\mathbf{m}^{\mathbf{2}}\right)\end{array}$ \\
\hline 1 & 427.11 & $1.8242 \times 10^{-7}$ & $6.7577 \times 10^{-8}$ \\
2 & 177.11 & $1.5144 \times 10^{-7}$ & $9.8563 \times 10^{-8}$ \\
3 & 93.77 & $1.2044 \times 10^{-7}$ & $1.2956 \times 10^{-7}$ \\
4 & 52.11 & $8.9464 \times 10^{-8}$ & $1.6054 \times 10^{-7}$ \\
5 & 27.11 & $5.8478 \times 10^{-8}$ & $1.9152 \times 10^{-7}$ \\
6 & 10.44 & $2.7483 \times 10^{-8}$ & $2.2252 \times 10^{-7}$ \\
\hline
\end{tabular}

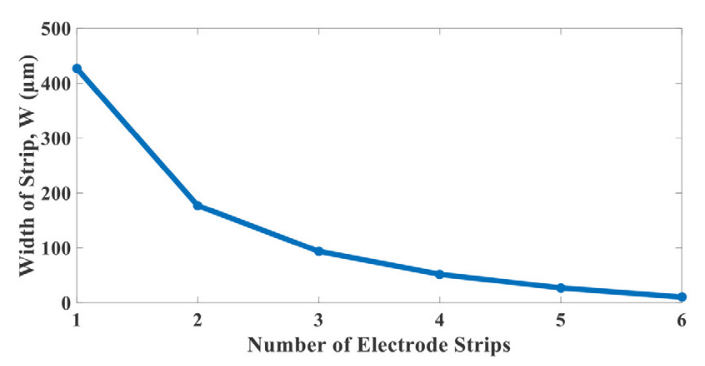

(a)

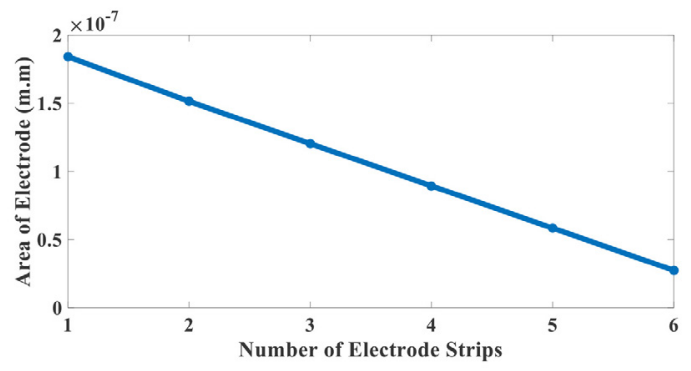

(b)

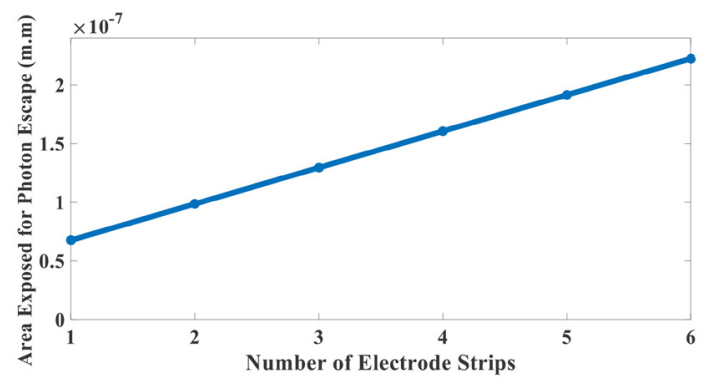

(c)

Figure 4: Comparison for (a) width of strip for each number of strips in the fork design, (b) area of electrode for every number of stripe and (c) area exposed for photon escape

by the electrode. The design with biggest area exposed for photon to escape enables more photon to escape LED chip to free region. This increases the extraction efficiency of the LED.

In summary, 6 strips design is the most optimum. The design has the most area for photons produced in active region to escape without reducing the area of effective current density spreading. Uniform current density in ITO leads to a more uniform current density in active region.
This research provide theoretical proof on how the extraction efficiency can be increased to be more than $50 \%$. However, to further validate the calculation, ray tracing optical simulation could be done in the future to simulate a large number of photons generated in the active region to escape the chip. The bottom layer of the LED can be coated with thin layer of silver as it has 99\% reflectivity thus can reflect rays generated towards the bottom to be reflected upwards. Enhancing the extraction efficiency would be a huge contribution in this field where LED is desired to have high extraction efficiency. 


\section{Conclusion}

Effective current spreading length, for Indium Tin Oxide (ITO) of thickness $300 \mathrm{~nm}$ is known, which is at $36.44 \mu \mathrm{m}$. The value of is then used to find the most suitable electrode pattern using the 'fork' design. value is used to find the distance between electrode 'fingers' and distance between electrode and edge of LED dice. The most suitable design is fork design with 6 fingers. The design gives minimum electrode contact area with ITO and maximum area exposed for photon to escape, making the design has least number of photon produced in active region to be absorbed by the electrode. This research provided an analysis on the design of electrode and how to optimize the design to provide better extraction efficiency. This theoretical work will provide solutions to improve the design of LED with sufficient amount of data.

\section{Acknowledgements}

This work was supported by Department of Physics, International Islamic University Malaysia, Malaysia. This research work has been supported by Fundamental Research Grant Scheme 19-033-0641.

\section{References}

Hao, G.-D., Taniguchi, M., Tamari, N., \& Inoue, S. I. (2016). Enhanced wall-plug efficiency in AlGaN-based deep-ultraviolet lightemitting diodes with uniform current spreading p-electrode structures. Journal of Physics D-Applied Physics, 49(23), 235101. doi:10.1088/0022-3727/49/23/235101

Huang, S. J., et al., Huang, S., Fan, B., Chen, Z., Zheng, Z., Luo, H., Wu, Z., Wang, G., Jiang, H. (2013). Lateral current spreading effect on the efficiency droop in GaN-based light-emitting diodes. Journal of Display Technology, 9(4), 266-271. doi:10.1109/ jdt.2012.2225092

Huh, C., Lee, J.-M., Kim, D.-J., \& Park, S.J. (2002). Improvement in light-output efficiency of $\mathrm{InGaN} / \mathrm{GaN}$ multiple- quantum well light-emitting diodes by current blocking layer. Journal of Applied Physics, 92(5), 2248-2250. doi:10.1063/1.1497467

Kim, H., Kim, K.-K., Choi, K.-K., Kim, H., Song, J.-O., Cho, J., Baik, K. H., Sone, C., \& Park, Y. (2007). Design of highefficiency GaN-based light emitting diodes with vertical injection geometry. Applied Physics Letters, 91(2), 023510. doi:10.1063/1.2756139

Li, C.-K., \& Wu, Y.-R. (2012). Study on the current spreading effect and light extraction enhancement of vertical GaN/InGaN LEDs. IEEE Transactions on Electron Devices, 59(2): p. 400-407. doi:10.1109/ted.2011.2176132

Ryu, H.-Y., \& Shim, J.-I. (2011). Effect of current spreading on the efficiency droop of InGaN light-emitting diodes. Optics Express, 19(4), 2886-2894. doi:10.1364/oe.19.002886

Schubert, E. F., Cho, J., \& Kim, J. K. (2018). Light-emitting diodes. https://doi. org/10.1002/0471238961.12090708110919 08.a01.pub3

Wang, P., Wei, W., Cao, B., Gan, Z., \& Liu, S. (2010). Simulation of current spreading for GaN-based light-emitting diodes. Optics \& Laser Technology, 42(5), p. 737-740. doi:10.1016/j.optlastec.2009.11.018

Yun, J. S., Shim, J. I., \& Shin, D. S. (2009). Enhancing current spreading by simple electrode pattern design methodology in lateral $\mathrm{GaN} / \mathrm{InGaN}$ LEDs. Electronics Letters, 45(13), 703-705. doi:10.1049/ el.2009.0803

Zhou, S., Wang, S., Liu, S., \& Ding, H. (2013). High power GaN-based LEDs with low optical loss electrode structure. Optics \& Laser Technology, 54, 321325. doi:10.1016/j.optlastec.2013.06.017

Zhou, S., Yuan, S., Liu, Y., Guo, L. J., Liu, S., \& Ding, H. (2015). Highly efficient and reliable high power LEDs with patterned sapphire substrate and strip-shaped 
distributed current blocking layer. Applied Surface Science, 355, 1013-1019. doi:10.1016/j.apsusc.2015.07.194

Zhou, S., Zheng, C., Lv, J., Gao, Y., Wang, R., \& Liu, S. (2017). GaN-based flip-chip LEDs with highly reflective ITO/DBR p-type and via hole-based n-type contacts for enhanced current spreading and light extraction. Optics \& Laser Technology, 92, 95-100. doi:10.1016/j.optlastec.2017.01.017
Zhou, S., Liu, X., Gao, Y., Liu, Y., Liu, M., Liu, Z., Gui, C., \& Liu, S. (2017). Numerical and experimental investigation of $\mathrm{GaN}$ based flip-chip light-emitting diodes with highly reflective $\mathrm{Ag} / \mathrm{TiW}$ and ITO/DBR Ohmic contacts. Optics Express, 25(22), 26615.

Zhou, S., Liu, X., Yan, H., Chen, Z., Liu, Y., \& Liu, S. (2019). Highly efficient GaNbased high-power flip-chip light-emitting diodes. Optics express, 27(12), A669-A692. 